\title{
High-dose-rate brachytherapy in the treatment of skin Kaposi sarcoma
}

\author{
Maria Ángeles González Ruiz, MD!, Juan Quirós Rivero, MD!', Julia Luisa Muñoz Garcia, MD!, \\ Joaquín José Cabrera Rodríguez, MD', Yesika Ríos Kavadoy, MD', María Francisca Ropero Carmona, MD', \\ Almudena Corbacho Campos, MD!, Fernando García Urra, MD!, María Cristina Cruz Muñoz, MD!, \\ Amanda Ruiz Herrero, MD!, Pedro Almendral Manzano, PhD² \\ 'Department of Radiation Oncology, Infanta Cristina Hospital, Badajoz, ${ }^{2}$ Department of Radiation Physics, Infanta Cristina Hospital, Badajoz, Spain
}

\begin{abstract}
Purpose: The aim of the study is to review our experience in treatment of Kaposi sarcoma (KS) lesions with highdose-rate (HDR) brachytherapy.

Material and methods: We present five new KS lesions (three patients) that were treated in our hospital from May 2016 to February 2017 with HDR brachytherapy using Valencia applicators. The treatment was delivered in 5 Gy fractions over five sessions, on alternate days. Total dose of $25 \mathrm{~Gy}\left(\mathrm{EQD}_{2} 31.25 \mathrm{~Gy}\right)$ was delivered. All patients were male, Caucasian, without a history of HIV, organ transplantation, or current immunosuppressive therapy. The median age was 76 years.

Results: All lesions (100\%) were located in lower limbs (60\% in the ankle, 20\% in the leg, and 20\% in the foot), and their development was progressive. No lesion was greater than $2 \mathrm{~cm}$ (range, $0.5-1.5 \mathrm{~cm}$ ). With a median follow-up of 15 months, all patients had a complete response to the treatment, with no evidence of local recurrence or tumor progression. Most of the patients $(80 \%)$ had no acute toxicity; only one patient developed erythema grade 2.

Conclusions: HDR brachytherapy could be a good option of treatment for these types of lesions, especially in elderly patients, or when cosmetic results are not good after surgery. Brachytherapy with the Valencia applicator, using hypofractionated regimen provides excellent results in terms of cosmetic and local control, and furthermore, facilitates treatment compliance, which is very relevant in elderly patients. HDR brachytherapy offers a simple, safe, quick, and attractive non-surgical treatment option.

Key words: brachytherapy, Kaposi sarcoma, skin cancer, Valencia applicator.

\section{Purpose}

Kaposi sarcoma (KS) is a multi-focal, angioproliferative neoplasm that usually appears on the skin, but can also involve the visceral organs. This disease was described for the first time by Moritz Kaposi in 1872 [1]. It is an endemic disease in Africa; however, nowadays, is highly related to immunosuppressive states, especially to human herpes virus 8 (HHV-8) [2]. The world prevalence is between 2-8\%, being up to $50 \%$ in Africans. Kaposi sarcoma development always occurs when the immune system is depressed, and it can be developed in four different clinical forms: 1) classic: cutaneous form, especially localized in lower limbs; 2) endemic: prevalent in Africa, similar to classic form, but more aggressive in younger patients; 3 ) post-transplant: in relationship with the immunosuppressive state; 4) human immunodeficiency virus (HIV)-related (epidemic KS).

The clinical evolution of KS is extremely variable. Most often, it follows a benign course and, although progressive, tends to remain confined to the skin and sub- cutaneous tissues. The skin lesions are purplish, reddish blue, or dark brown/black macules, plaques or nodules that involve skin and mucous membranes. These lesions may bleed, ulcerate, and may be associated with lymphedema, pain, and secondary infection. Most patients with KS have a long and indolent course of the disease [3].

For local disease, there is no consensus on the optimal tumor-directed therapy. Although many active treatments have been described (radiation therapy, excision, cryotherapy, laser ablation, intralesional, or topical therapy), therapeutic choices are often made based on the experience and medical discipline of the clinician, and also, on the preferences of the patients and their comorbidities. Since KS lesions are considered very radiosensitive, the most widely advocated type of treatment with excellent local control has been radiotherapy. For more aggressive and extensive diseases, or when radiotherapy is not available, chemotherapy has been successfully employed. Since the disease usually appears with solitary lesions, 
the most frequent approaches used are local irradiation with low-energy electrons or superficial X-rays.

The advantages of high-dose-rate (HDR) brachytherapy to treat $\mathrm{KS}$ are better dose distribution and better protection of subcutaneous tissues near the tumor, compared to external beam radiation therapy (EBRT). HDR brachytherapy is considered superior to EBRT in terms of dosimetric coverage that is related to lower complication rates. In order to choose between these two techniques, characteristics of the patient, Karfnosky performance status (KPS), and patient's delivery comfort would be considered. Furthermore, the radiation oncologist's availability to deliver either modality of treatment needs to be considered as well.

In our department, we choose HDR brachytherapy based on comfort of patients, lesser number of fractions, reduced healthcare costs, and on minimizing medical EBRT waiting lists. Although several studies describe this technique, there is little of clinical outcome data published.

The following study reviews our experience with high-dose-rate (HDR) brachytherapy and hypofractionated schedule in five classic KS lesions.

\section{Material and methods}

\section{Research tool}

In Pubmed and Database, we introduced the following terms to search for different trials of this method of treatment: 'brachytherapy', Kaposi sarcoma', 'skin cancer', and 'radiotherapy'.

\section{Patients}

From May 2016 to February 2017, 3 patients with 5 skin KS lesions were treated with HDR brachytherapy with Valencia applicators (Nucletron, an Elekta company, Elekta AB, Stockholm, Sweden) in our Department. Patients enrolled in this study had KS lesions confirmed by biopsy, located anywhere on the body, and all of them were superficial skin lesions ( $<3 \mathrm{~mm}$ thickness) and $<2 \mathrm{~cm}$ in size. Patients who had multiple lesions were treated concurrently. Data were collected retrospectively and all patients gave informed consent.

The HDR plesiotherapy is administered by a microSelectron HDR brachytherapy ${ }^{192}$ Ir system (Nucletron, an Elekta company, Elekta AB, Stockholm, Sweden).

General indications for HDR plesiotherapy:

1. age $>18$ years-old,

2. radical treatment of superficial nonmelanoma skin cancers (NMSCs) T1-2 after biopsy,

3. size of the lesion $\leq 2 \mathrm{~cm}$ to use Valencia applicators,

4. size of the lesion $>2 \mathrm{~cm}$ to use hand-made custom molds,

5. any location,

6. thickness of the lesion $<0.5 \mathrm{~cm}$,

7. no vascular diseases (high-risk of ulceration),

8. surgery unfit,

9. adjuvant treatment of excised lesions (T1-2) with close or positive surgical margins,

10. treatment of recurrent tumors,

11. palliative treatment for multiple and diffuse lesions in systemic disease.

\section{Protocols}

The gross tumor volume (GTV) was generally assessed visually or by dermatoscope. The planning target volume (PTV) included a $0.5-1 \mathrm{~cm}$ safety margin around the GTV. The safety margin was chosen depending on the size of the lesion (lesion < $2 \mathrm{~cm}$ : margin $0.5 \mathrm{~cm}$; lesion $\geq 2 \mathrm{~cm}$ : margin $1 \mathrm{~cm}$ ). The appropriate Valencia applicator diameter (2 or $3 \mathrm{~cm}$ ) was chosen depending on the size of the PTV. Lesions bigger than $2 \mathrm{~cm}$ are not treated with these applicators; for them, we use hand-made custom molds. All the lesions were superficial, without evidence of significant thickness and evaluated by physical exam. Usually, for superficial lesions, we don't use image method to evaluate their depth.

The treatment dose was prescribed 3-4 $\mathrm{mm}$ from the surface of the skin lesions. High accuracy of tumor depth measurement was critical due to the high dose gradient. The prescription dose was 25 Gy in 5 fractions, delivered two/three times a week, with a minimum interval of 48 hours between fractions, and not exceeding 16 days. For biological effective dose (BED) calculations and equivalent dose to $2 \mathrm{~Gy}\left(\mathrm{EQD}_{2}\right)$, the linear quadratic model was used with an $\alpha / \beta$ of 10 (BED $~ 37.5 \mathrm{~Gy}$; EQD 2 : 31.25 Gy).

Immobilization was achieved in most cases using tape, or an articulated arm device provided by Elekta. Skin marker delineating the outside applicator circumference was used to ensure reproducible treatment conditions. All treatments were controlled by the radiation oncologist to ensure the positioning of the applicator. Patients were instructed to avoid using moisturizer before the treatment (Figures 1 and 2).

\section{Technique and treatment planning}

The treatment is administered with a microSelectronHDR brachytherapy ${ }^{192}$ Ir system and Valencia applicators (Elekta AB, Stockholm, Sweden). In this applicator, the source is positioned at the center of a conical aperture, surrounded by a tungsten shielding that limit the irradiation to the required area, with a source-skin distance of approximately $16 \mathrm{~mm}$. The design also includes a flattening filter to ensure the uniformity of the dose in a plane perpendicular to the axis of the applicator, and a plastic cap to absorb the scattered electrons produced by the interaction of the radiation in the shielding and the flattening filter. Two applicator sizes are available $\mathrm{H} 2$ and $\mathrm{H} 3$, with diameter of the treatment area equal to 2 and $3 \mathrm{~cm}$, respectively.

Our treatment planning system does not allow to calculate the dose distribution from Valencia applicators. Therefore, we assume water-equivalent tissue and calculate the treatment time with a spreadsheet, based on the dosimetric data obtained from Monte Carlo simulation by Granero et al. [4], i.e., output factors (dose rate at $3 \mathrm{~mm}$ depth per unit Kerma strength) and percentage of depth dose. The applicator diameter and the prescription depth ( 3 or $4 \mathrm{~mm}$ ) are decided by the radiation oncologist after physical examination.

\section{End points}

The end points of this study are to analyze the efficacy, safety, and cosmetic outcomes of the brachytherapy 


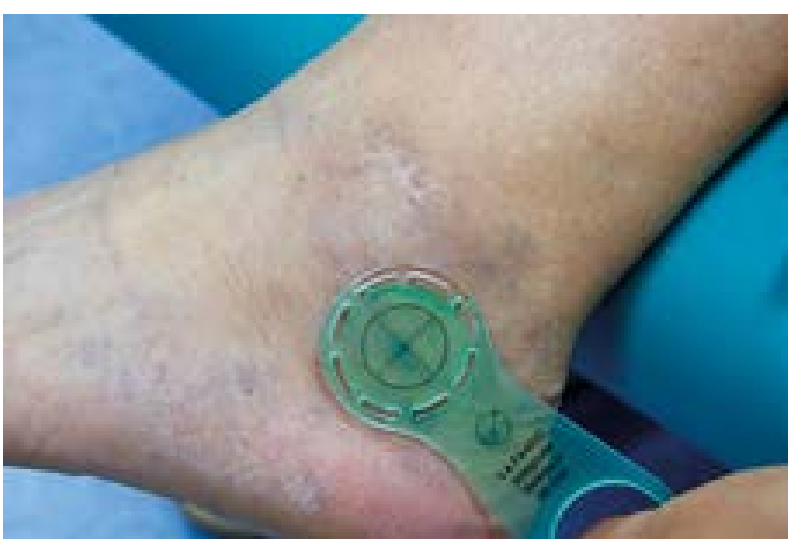

Fig. 1. Valencia applicator template of $2 \mathrm{~cm}$. The outer discontinuous fenestrations help to delimit with a marker the exact position of the Valencia applicator

treatment, using the Valencia applicators with a hypofractionated schedule. Follow-up was scheduled on every three months during the first year. A complete response was defined as the complete resolution of the palpable tumor, and a partial response was defined as a reduction in palpable tumor to less than $50 \%$ of the pre-treatment area. Recurrence was defined as any skin progression. Adverse events for acute and late skin toxicity were evaluated using Common Terminology Criteria Adverse Events (CTCAE v. 4.0). Patients were also asked about post-treatment satisfaction in terms of cosmetic results.

Cosmesis was scored as excellent, good, fair, or poor, based on the presence or absence of edema, alopecia, pigment changes, telangiectasia, skin fibrosis, and skin atrophy or ulceration [5] (Table 1).

SPSS Statistics program was used for statistical analysis. Survival rates, disease free survival (DFS), and local control (LC) were estimated with Kaplan-Meier curves.

\section{Results}

From May 2016 to February 2017, five new KS lesions (three patients) were treated in our hospital. All patients were male, Caucasian without a history of HIV, organ transplantation, or current immunosuppressive therapy. The median age was 76 years old at the time their respective lesions were treated (range, 73-78) (Table 2).

All lesions (100\%) were in lower limbs $(60 \%$ in the an$\mathrm{kle}, 20 \%$ in the leg and $20 \%$ in the foot), and their development was progressive. No lesion was greater than $2 \mathrm{~cm}$ (range, $0.5-2 \mathrm{~cm}$ ). One patient $(33 \%)$ had thrombophlebitis related to lesion, and no patients had pain or bleeding.

$\mathrm{H} 2$ Valencia applicator was used in $80 \%$, and H3 Valencia applicator was applied in $20 \%$ of lesions. Treatment was

Table 1. Cosmetic results

\begin{tabular}{ll} 
Excellent & With no defects \\
\hline Good & Minor defects (pigment changes or/and alopecia) \\
\hline Fair & $\begin{array}{l}\text { Significant defects (pigment changes or/and } \\
\text { alopecia, or/and edema, or/and telangiectasia) }\end{array}$ \\
\hline Poor & Ulceration
\end{tabular}

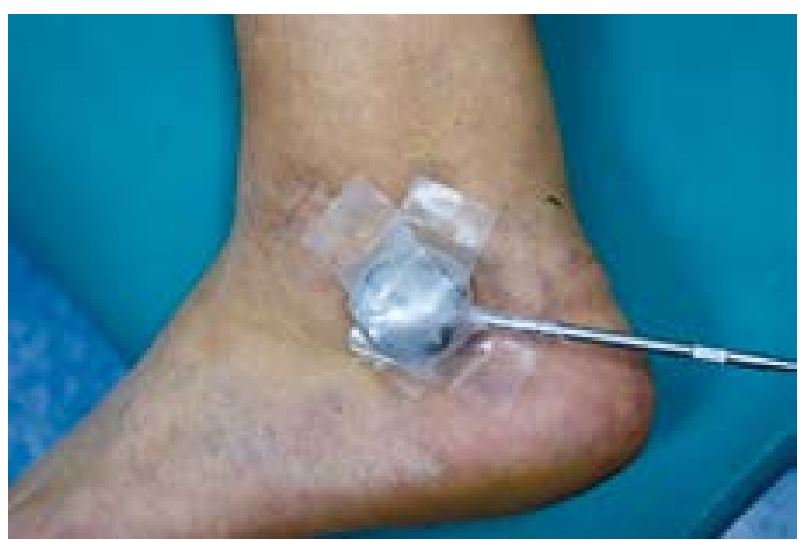

Fig. 2. Valencia applicator with $2 \mathrm{~cm}$ of diameter (H2). Fixation system with tape

delivered in 5 Gy fractions over five sessions, on alternate days. Total dose delivered was $25 \mathrm{~Gy}\left(\mathrm{EQD}_{2} 31.25 \mathrm{~Gy}\right)$.

With a median follow-up of 18 months (range, 9-18), all patients had a complete response to treatment, with no evidence of local recurrence or tumor progression (DFS and LC 100\%) (Figures 3-5).

Most of the patients $(80 \%)$ had no acute toxicity; only one patient developed erythema grade 2 . No patients had late adverse events. Cosmetic results were excellent in all the lesions treated, and cosmetic satisfaction of the patients was very satisfied.

\section{Discussion}

Classic KS occurs most often in Mediterranean or Central/Eastern European elderly men. It appears between the fifth and seventh decade of life, and it is more common in males than in females $(15: 1)$. The most frequent locations are the lower limbs with cutaneous affectation and centripetal extension [6].

Table 2. Patients characteristics

\begin{tabular}{lc} 
Factor & Frequency \\
\hline Sex & $100 \%$ \\
\hline Males & $0 \%$ \\
\hline Memales & $76(73-78)$ \\
\hline KPS & $0(100 \%)$ \\
\hline Location & $60 \%$ \\
\hline Ankle & $20 \%$ \\
\hline Foot & $20 \%$ \\
\hline Dose fractionation & 25 Gy in 5 fractions (EQD 231.25 Gy) \\
\hline Applicators used & $80 \%$ \\
\hline H2 Valencia & $20 \%$ \\
\hline H3 Valencia & \\
\hline
\end{tabular}




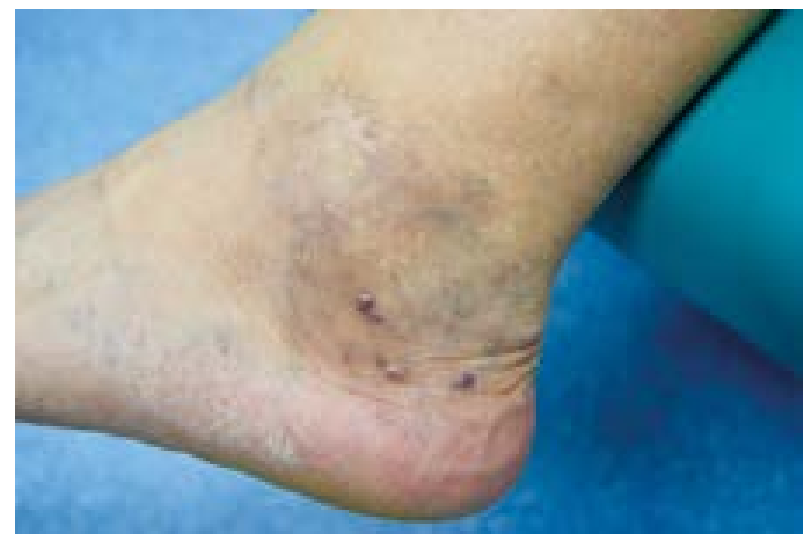

Fig. 3. Three superficial Kaposi sarcoma lesions of $1 \mathrm{~cm}$ localized in the ankle

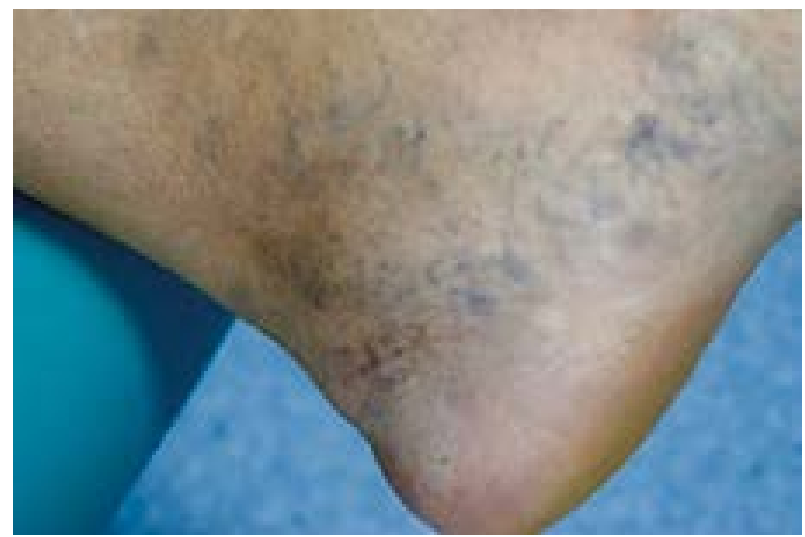

Fig. 5. Six months post-treatment results. Resolution of the Kaposi sarcoma lesion

Due to the absence of treatments capable of eradicating latent HHV-8 infection, the treatment for classic KS is mainly palliative. Different methods with different responses have been applied to classic KS. These methods include single or multiple drug chemotherapy, electrodes-

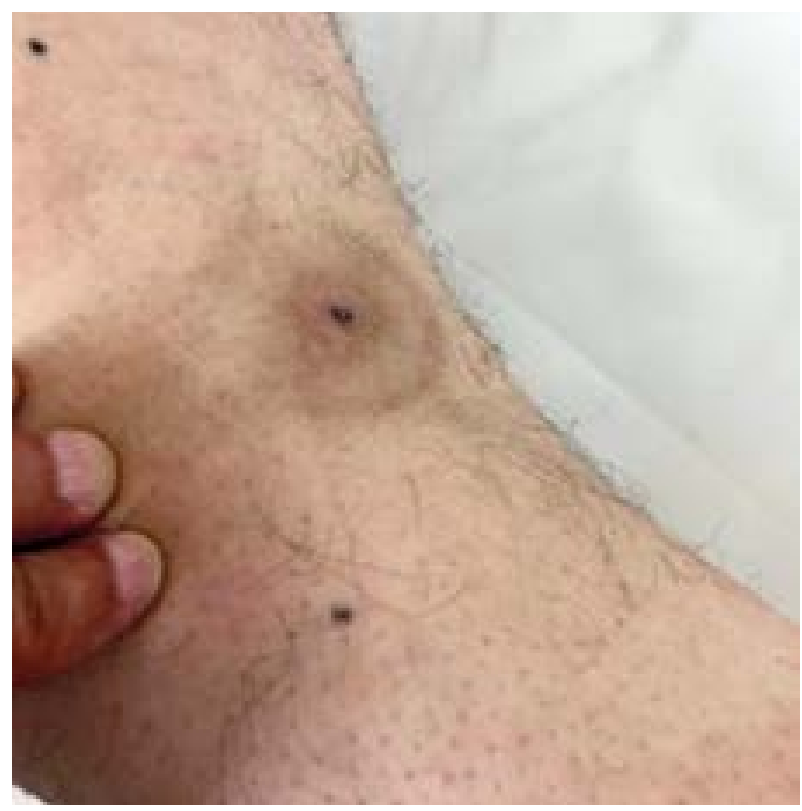

Fig. 4. Three months post-treatment results. Regression of the Kaposi sarcoma lesion

sication and curettage, cryotherapy, surgical excision, and immunotherapy [7].

Radiation therapy has a long history as an effective local therapy for Kaposi sarcoma. Several successful strategies have been described [8-13] (Table 3), and high rates of tumor regression have been reported using different energy sources (Cobalt-60, low-energy electrons, superficial X-rays). There is an important difference in doses (6 to $60 \mathrm{~Gy}$ ) and fractionation schedules (doses from 6 to 12 Gy in single fractions, or larger total doses administered in smaller fractions during several weeks).

Although no clear consensus on a standard treatment regimen exists, the high level of radiosensitivity of KS is well established, and excellent results have been obtained with doses of 20-30 Gy delivered over 2-3 weeks.

Table 3. Retrospective trials of radiotherapy treatment for Kaposi sarcoma

\begin{tabular}{|c|c|c|c|c|c|c|}
\hline Author & Technique & $n$ & $\begin{array}{c}\text { Fraction dose } \\
\text { (Gy) }\end{array}$ & Fractions & $\begin{array}{l}\text { Response } \\
\text { rate }\end{array}$ & Side effects \\
\hline Nisce, et al. [8] & TSEB/SSEG & 20 & 4 & $6-8$ & $85 \% C R$ & - \\
\hline Tsao, et al. [9] & EBT & 17 & $4-8^{*}$ & $1-10$ & $30 \% \mathrm{CR}$ & $\begin{array}{l}\text { Dry desquamation hyperpigmentation } \\
\text { lymphedema }\end{array}$ \\
\hline Yildiz, et al. [10] & EBT & 12 & 8 & 1 & $89,7 \%$ CR & Hyperpigmentation fibrosis \\
\hline Hauerstock, et al. [11] & EBT & 16 & 2 & 15 & $88 \% C R$ & Dermatitis \\
\hline Kirova, et al. [12] & EBT & 643 & 2.5 & 12 & $92 \% C R$ & Dermatitis \\
\hline Hamilton, et al. [13] & EBT & 91 & $8^{* \star}$ & 1 & $\begin{array}{l}70 \% \text { CR EF } \\
39 \% \text { CR LF }\end{array}$ & - \\
\hline Evans, et al. [14] & HDR BT & 16 & $8-20$ & 1 & - & $>20$ Gy moderate-severe desquamation \\
\hline Syndikus, et al. [15] $]^{\star * *}$ & HDR BT & 6 & 8 & 3 & $100 \%$ CR & Mucositis \\
\hline
\end{tabular}

TSEB - total skin electron beam therapy, SSEB-subtotal skin electron beam therapy, CR-complete response, EBT-external beam therapy, EF-extended field, LF-local field *8 Gy in 1 fraction, or 20 Gy in 5 daily fractions (most frequent scheme), **8 Gy in 1 fraction (most frequent scheme), ${ }^{* * *}$ palate Kaposi sarcoma 
Nowadays, new methods of radiotherapy have been employed for these types of lesions, and high-dose brachytherapy using a skin surface applicator is one of them [14, 15]. This treatment seems to be an effective, non-invasive option for patients with non-melanomatous skin cancers $[16,17]$.

Kasper et al. published the only report with KS skin lesion treated with HDR plesiotherapy. Their results report sixteen patients treated in four to six fractions with Leipzig applicator, over a period of approximately 12 days, with a dose ranged from 24 to $35 \mathrm{~Gy}\left(\mathrm{EQD}_{2} 28\right.$ $43.75 \mathrm{~Gy})$. All of the patients had a complete response after 2 years of follow-up [18].

Our study is the first to report on clinical outcomes with Valencia applicators in KS skin lesions. The design of these applicators is similar to Leipzig applicators, with the addition of a flattening filter to improve the dose profiles and to limit the penumbra. These changes allow the radiation to focus on the target volume, while irradiation of normal tissues is minimal [19].

In this study, we applied the hypofractionated schedule of 25 Gy in 5 fractions (EQD 31.5 Gy) delivered twothree times a week, and the results are excellent with a complete response in all the lesions treated.

Traditionally in our department, KS skin lesions were treated with 6-MeV electrons with a dose ranged from 20 to 30 Gy in 5-10 fractions (3-4 Gy/fraction; $\mathrm{EQD}_{2}$ : 23.33-32.5 Gy) with an excellent local control. HDR brachytherapy for non-melanomatous skin cancers was introduced in our department in June 2015, and because of the quick acceptance and good results of the treatment for skin cancers, we began to use it also for KS lesions. Furthermore, it is a comfortable technique that reduces the number of treatment fractions, minimizing healthcare costs and EBRT waiting lists.

\section{Conclusions}

HDR brachytherapy treatment seems to be an effective and non-invasive option for patients with small cutaneous KS, delivering excellent cosmesis and local control in our small series.

It is a technique highly recommended in elderly patients and when cosmetic results are not good after the surgery. Furthermore, it is a good option when the patients refuse surgery, or when this option is not possible because of their comorbidities. The complication rate of brachytherapy is acceptable and the costs are low. In spite of all these advantages, more clinical studies with larger numbers of patients and longer follow-up are needed to confirm this method as an excellent option of treatment for many patients.

\section{Acknowledgement}

This is to express the gratitude toward Dr. Quirós for his great help and involvement.

\section{Disclosure}

Autohors report no conflict of interest.

\section{References}

1. Kaposi M. Idiopathisches multiples pigmentsarcom der haut. Arch Dermatol Syphilol 1872; 4: 265-273.

2. Gao SJ, Kingsley L, Hoover DR et al. Seroconversion to antibodies against Kaposi's sarcoma-associated herpesvirus-related latent nuclear antigens before the development of Kaposi's sarcoma. N Engl J Med 1996; 335: 233-241.

3. Bhutani M, Polizzotto MN, Uldrick TS et al. Kaposi sarcomaassociated herpesvirus-associated malignancies: epidemiology, pathogenesis, and advances in treatment. Semin Oncol 2015; 42: 223-246.

4. Granero D, Pérez-Calatayud J, Gimeno J et al. Design and evaluation of a HDR skin applicator with flattening filter. Med Phys 2008; 35: 495-503.

5. Arenas M, Sabater S, Hernández V et al. Cosmetic outcome of breast conservative treatment for early stage breast cancer. Clin Transl Oncol 2006; 8: 334-338.

6. Tappero JW, Conant MA, Wolfe SF et al. Kaposi's sarcoma: epidemiology, pathogenesis, histology, clinical spectrum, staging criteria, and therapy. J Am Acad Dermatol 1993; 28: 371-395.

7. Régnier-Rosencher E, Guillot B, Dulpin N. Treatments for classic Kaposi sarcoma: a systematic review of the literature. J Am Acad Dermatol 2013; 68: 313-331.

8. Nisce LZ, Safai B, Poussin-Rosillo H. Once weekly total and subtotal skin electron beam therapy for Kaposi's sarcoma. Cancer 1981; 47: 640-644.

9. Tsao MN, Sinclair E, Assaad D et al. Radiation therapy for the treatment of skin Kaposi sarcoma. Ann Palliat Med 2016; 5: 298-302.

10. Yildiz F, Ozyar E, Uzal D et al. Kaposi's sarcoma: the efficacy of a single fraction of 800 cGy. Dermatology 1997; 195: 142-144.

11. Hauerstock D, Gerstein W, Vuong T. Results of radiation therapy for treatment of classic Kaposi sarcoma. J Cutan Med Surg 2009; 13: 18.

12. Kirova YM, Belembaogo E, Frikha $\mathrm{H}$ et al. Radiotherapy in the management of epidemic Kaposi's sarcoma: a retrospective study of 643 cases. Radiother Oncol 1998; 46: 19-22.

13. Hamilton CR, Cummings BJ, Harwood AR. Radiotherapy of Kaposi's sarcoma. Int J Radiat Oncol Biol Phys 1986; 12: 19311935.

14. Evans MD, Yassa M, Podgorsak EB et al. Surface applicators for high-dose-rate brachytherapy in aids-related Kaposi's sarcoma. Int J Radiat Oncol Biol Phys 1997; 39: 769-774.

15. Syndikus I, Vinall A, Rogers P et al. High dose rate Microselectron moulds for Kaposi sarcoma of the palate. Radiother Oncol 1997; 42:167-170.

16. Skowronek J. Brachytherapy in the treatment of skin cancer: an overview. Postep Derm Alergol 2015; 32: 362-367.

17. Delishaj D, Rembielak A, Manfredi B et al. Non-melanoma skin cancer treated with high-dose-rate brachytherapy: a review of literature. J Contemp Brachytherapy 2016; 8: 533-540.

18. Kasper ME, Richter $S$, Warren $N$ et al. Complete response of endemic Kaposi sarcoma lesions with high-dose-rate brachytherapy: treatment method, results, and toxicity using skin surface applicators. Brachytherapy 2013; 12: 495-499.

19. Granero D, Pérez-Calatayud J, Ballester F. Radiation leakage study for the Valencia applicators. Med Phys 2013; 29: 60-64. 